\title{
A NECESSARY CONDITION FOR TWO STRING LINKS TO HAVE THE SAME CLOSURE UP TO CONCORDANCE
}

\author{
José Eduardo Prado Pires de Campos
}

\begin{abstract}
To a link (obtained by closing a string link) we associate a certain diagram of groups. If two links are concordant, we show that there exists a certain type of isomorphism between the group diagrams.
\end{abstract}

\section{Introduction}

The concept of knot concordance (or knot cobordism) was introduced by Fox and Milnor [5]. Its study, as well as the study of link concordance, continued in the works of Cappell and Shaneson [3] Tristram [15], Levine ([9], [10]), Ko [8] and others.

In this paper we study link concordance via string links. Le Dimet [4] introduced the group of cobordism classes of $k$-string links (a string link is a generalization of a braid-see Definition 1 below). Besides their own interest, $k$-string links are naturally related to links because one obtains a $k$-link by simply closing a $k$-string link, like one does for a braid, with the advantage that the number of strings is preserved. The group of concordance classes of $k$-string links also have a kind of Artin representation (see [6]). This allows to break the problem of studying links in two problems: studying $k$-string links (with their natural multiplication) and studying when two $k$-string links have the same closure. This string-link approach was shown to be very useful in the case of link-homotopy allowing Habegger and Lin [7] to classify links up to linkhomotopy. With a similar approach, Habegger and Lin [6] also obtained some advances in the case of link concordance, but, in their own words, "that was far from leading to a classification of links up to concordance".

Previously, Levine [11] had studied link-homotopy using an approach based on peripheral invariants, similar to that of Waldhausen's Theorem for links up to ambient isotopy (see [16]). In our paper [1] we provided a connection between the two approaches, obtaining a certain diagram of groups that distinguishes links

Mathematics Subject Classification. 57M25.

Key words and phrases. Link, string link, link concordance.

Received June 12, 2014; revised March 3, 2015. 
if and only if they are not link-homotopic. The diagram had a small mistake that we correct in section 4 below.

In this paper we consider similar results for the case of link concordance. In this case, we show only that if two links are link concordant then there exists a certain type of isomorphism between the associated group diagrams, thus providing a necessary algebraic condition for two string links to have concordant closures.

This paper is divided as follows: in section 2, we deal with Habegger-Lin's actions for string-links (up to ambient isotopy), where we associate certain epimorphisms to elements in the stabilizer of 1 for both of Habegger-Lin's actions of $2 k$-string links on $k$-string links (see Fig. 2 for the actions). In section 3 , we study the case of link concordance, case in which both stabilizers coincide (see [6]) and we obtain a necessary condition for two string links to have the same closure up to concordance (Theorem 21 and Corollary 22). In section 4, we make a correction for the case of link-homotopy (as it appeared in our paper [1]). Our group diagram in [1] is a complete invariant, that is, it distinguishes links if and only if they are not link homotopic, but to show that we need a small change in the diagram that appeared in [1].

\section{Ambient isotopy}

We will use the following notation: $I$ is the interval $[0,1], D$ is the unit disk $\left\{x \in \mathbf{R}^{2} \mid\|x\| \leqslant 1\right\}, k \geqslant 1$ is an integer number, $\underline{k}$ is the set $\{1,2, \ldots, k\},(\forall i \in \underline{k})$ $a_{i}$ is the point $\left(-1+\frac{2 i}{k+1}, 0\right) \in D$ and $j_{0}: \underline{k} \times I \rightarrow D \times I$ is the map defined by $(i, x) j_{0}=\left(a_{i}, x\right)$. Note that, as above, if $f$ is a map, we will usually write (x) $f$ instead of $f(x)$.

DEFINITION 1. A $k$-string link is a (smooth or piecewise linear) proper embedding $f: \underline{k} \times I \rightarrow D \times I$ such that $\left.f\right|_{\underline{k} \times \partial I}=\left.j_{0}\right|_{\underline{k} \times \partial I}$ (see Fig. 1).

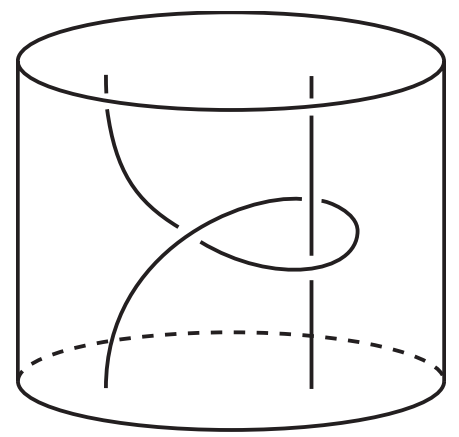

FIGURE 1. A 2-string link. 
The product of two $k$-string links $f$ and $g$, denoted by $f g$, is given by stacking $f$ on the top of $g$ and reparametrizing (see [1]). This product induces a monoid structure on the set $S L(k)$ of (ambient) isotopy classes of $k$-string links.

Habegger-Lin (see [6]) introduced a left and a right action of the monoid $S L(2 k)$ on the set $S L(k)$ that we will call Habegger-Lin's actions. We use a slightly different notation (see Fig. 2 below).

Definition 2. The reflection of a $k$-string link $f$ is the $k$-string link $f^{R}$ obtained by reflecting $f$ in $D \times \frac{1}{2}$.
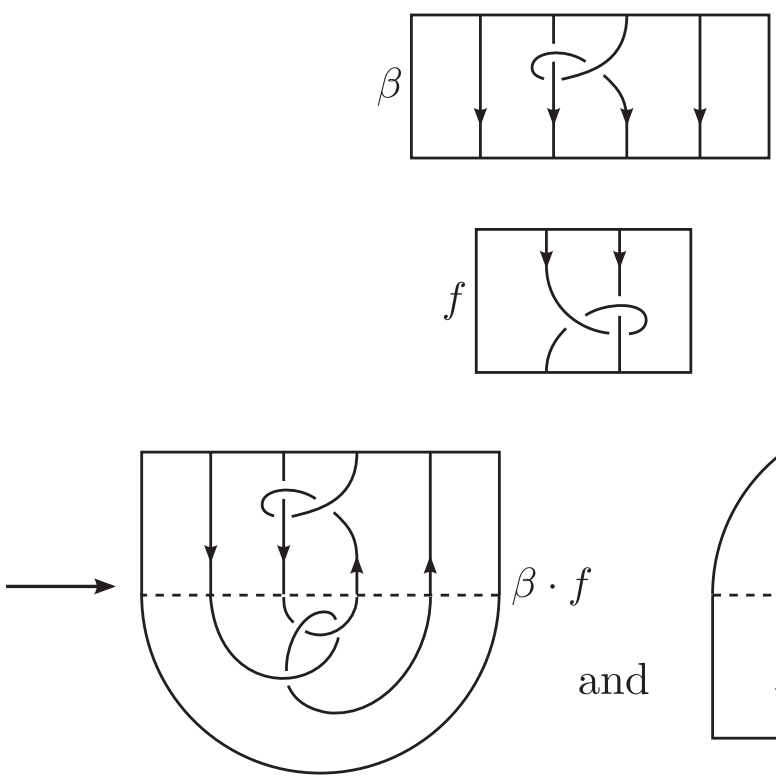

and

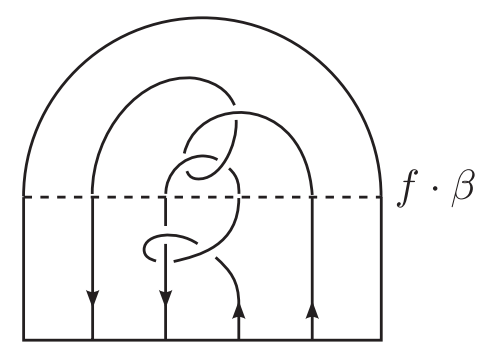

FIGURE 2. A 4-string link $\beta$ acts from the left and from the right on a 2-string link $f$.

Definition 3. A $k$-link (or a link of $k$ components) is an embedding of a disjoint union of ordered oriented circles $\bigsqcup_{i=1}^{k} S^{1}$ into $S^{3}$.

To a $k$-string link $f$ it is associated a $k$-link $\hat{f}$ called its closure (see [1]).

The fundamental group of the complement of a string link $f$ is called the group of $f$ and is denoted by $\pi(f)$.

For a group $G$, let $\left\{G_{n}\right\}, n \geqslant 1$, denote the lower central series of $G$, that is, $G_{1}=G$ and inductively $G_{n+1}=\left[G, G_{n}\right]$ (where for sets $A, B \subseteq G,[A, B]$ denotes the group generated by all commutators $[a, b]=a b a^{-1} b^{-1}, a \in A, b \in B$.) 
Let $\tilde{G}=\lim _{n} \frac{G}{G_{n}}$ be the nilpotent completion of $G$.

Let $F(k)$ denote the free group in $k$ generators $\alpha_{1}, \alpha_{2}, \ldots, \alpha_{k}$.

Let $f$ be a $k$-string link. We will denote by $x_{i}=x_{i}(f) \in \pi(f)$, for all $i \in \underline{k}$, the top meridians of $f$ and by $y_{i}=y_{i}(f) \in \pi(f)$, for all $i \in \underline{k}$, the bottom meridians of $f$ (see Fig. 3 and [2]).

For $j=0,1$, inclusions $i_{j}: D \times\{j\} \backslash \partial_{j} f \rightarrow D \times I \backslash f$ induce homomorphisms $\mu_{0}(f): F(k)=F\left(\alpha_{1}, \alpha_{2}, \ldots, \alpha_{k}\right) \rightarrow \pi(f), \quad\left(\alpha_{i}\right) \mu_{0}(f)=x_{i}(f), \quad$ and $\mu_{1}(f): F(k) \rightarrow$ $\pi(f),\left(\alpha_{i}\right) \mu_{1}(f)=y_{i}(f)$, called respectively, the top meridian map for $f$ and the bottom meridian map for $f$. By Stallings' Theorem [14] (see also [6]), they also induce isomorphisms on the lower central series quotients of fundamental groups:

$$
\frac{F(k)}{F(k)_{n}} \stackrel{\left(\mu_{0}(f)\right)_{n}}{\cong} \frac{\pi(f)}{\pi(f)_{n}} \stackrel{\left(\mu_{1}(f)\right)_{n}}{\cong} \frac{F(k)}{F(k)_{n}} .
$$

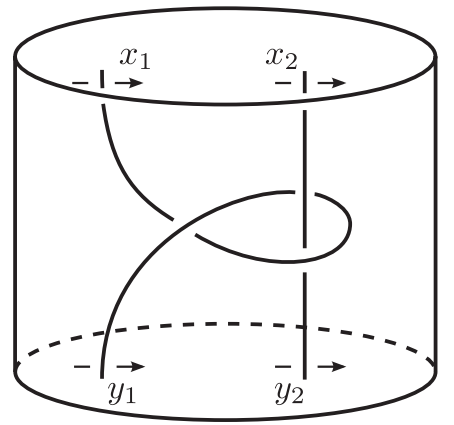

FiguRE 3. Top and bottom meridians of a 2-string link.

Therefore $\left(\mu_{0}(f)\right)_{n}\left(\mu_{1}(f)\right)_{n}^{-1}$ is an element $A_{n}(f) \in A u t\left(\frac{F(k)}{F(k)_{n}}\right)$, the group of automorphisms of $\frac{F(k)}{F(k)_{n}}$. Let us denote $A_{n}(f)$ also by $\bar{f}_{n}$.

$\mu_{0}(f)$ and $\mu_{1}(f)$ also induce isomorphisms (see [12]):

$$
\widetilde{F(k)} \underset{\mu_{0}(f)}{\cong} \widetilde{\pi(f)} \underset{\cong}{\stackrel{\mu_{1}(f)}{\longleftarrow} \widetilde{F(k)} .}
$$

Thus we have $A(f)=\tilde{f}=\widetilde{\mu_{0}(f)} \widetilde{\mu_{1}(f)} \tilde{\tilde{f}}^{-1} \in A u t(\widetilde{F(k)})$.

The associations $f \mapsto \overline{f_{n}}$ and $f \mapsto \tilde{f}$ are monoid homomorphism from $\operatorname{SL}(k)$ into $\operatorname{Aut}\left(\frac{F(k)}{F(k)_{n}}\right)$ and $\operatorname{Aut}(\widetilde{F(k)})$, respectively.

Note also that, since $\mu_{0}\left(f^{R}\right)=\mu_{1}(f)$ and $\mu_{1}\left(f^{R}\right)=\mu_{0}(f)$, we have $\left(\overline{f^{R}}\right)_{n}=$ $\bar{f}_{n}^{-1}$ and also $\widetilde{f^{R}}=\tilde{f}^{-1}$. 
Let $F(2 k)$ be the free group in $2 k$ generators $\alpha_{1}, \alpha_{2}, \ldots, \alpha_{k}, \widetilde{\alpha_{k}}, \ldots, \widetilde{\alpha_{2}}, \widetilde{\alpha_{1}}$ and $\left\langle\alpha_{i} \widetilde{\alpha}_{i}\right\rangle^{N}=\left\langle\alpha_{i} \widetilde{\alpha}_{i} \mid i \in \underline{k}\right\rangle^{N}$ be the normal subgroup of $F(2 k)$ generated by $\alpha_{i} \widetilde{\alpha}_{i}$, $i \in \underline{k}$. $i \in \underline{k}$.
Let $\left\langle\alpha_{i} \widetilde{\alpha}_{i} F(2 k)_{n}\right\rangle^{N}$ be the normal subgroup of $\frac{F(2 k)}{F(2 k)_{n}}$ generated by
$\alpha_{i} \widetilde{\alpha}_{i} F(2 k)_{n}, i \in \underline{k}$.

LEMMA 1. There is an isomorphism

$$
\lambda: \frac{F(2 k)}{\left\langle\alpha_{i}{\widetilde{\alpha_{i}}}^{N} F(2 k)_{n}\right.} \rightarrow \frac{\frac{F(2 k)}{F(2 k)_{n}}}{\left\langle\alpha_{i} \widetilde{\alpha}_{i} F(2 k)_{n}\right\rangle^{N}}
$$

defined by $\left(w\left\langle\alpha_{i} \widetilde{\alpha}_{i}\right\rangle^{N} F(2 k)_{n}\right) \lambda=w F(2 k)_{n}\left\langle\alpha_{i} \widetilde{\alpha}_{i} F(2 k)_{n}\right\rangle^{N}$, for any $w \in F(2 k)$.

Proof. First note that $w\left\langle\alpha_{i} \widetilde{\alpha}_{i}\right\rangle^{N} F(2 k)_{n}=\left\langle\alpha_{i} \widetilde{\alpha}_{i}\right\rangle^{N} F(2 k)_{n} \Leftrightarrow w \in\left\langle\alpha_{i} \widetilde{\alpha}_{i}\right\rangle^{N}$. $F(2 k)_{n} \Leftrightarrow w=u v \quad$ with $\quad u \in\left\langle\alpha_{i} \widetilde{\alpha}_{i}\right\rangle^{N}$ and $v \in F(2 k)_{n} \Leftrightarrow w F(2 k)_{n}=u v F(2 k)_{n}=$ $u F(2 k)_{n}$ with $u \in\left\langle\alpha_{i} \widetilde{\alpha}_{i}\right\rangle^{N} \Leftrightarrow w F(2 k)_{n} \in\left\langle\alpha_{i} \widetilde{\alpha}_{i} F(2 k)_{n}\right\rangle^{N}$.

It follows that, for any $w, w^{\prime} \in F(2 k)$, we have

$$
\begin{aligned}
w\left\langle\alpha_{i} \widetilde{\alpha}_{i}\right\rangle^{N} F(2 k)_{n} & =w^{\prime}\left\langle\alpha_{i} \widetilde{\alpha}_{i}\right\rangle^{N} F(2 k)_{n} \Leftrightarrow w^{-1} w^{\prime} \in\left\langle\alpha_{i} \widetilde{\alpha}_{i}\right\rangle^{N} F(2 k)_{n} \\
& \Leftrightarrow w^{-1} w^{\prime} F(2 k)_{n} \in\left\langle\alpha_{i} \tilde{\alpha}_{i} F(2 k)_{n}\right\rangle^{N} \\
& \Leftrightarrow\left(w^{-1} F(2 k)_{n}\right)\left(w^{\prime} F(2 k)_{n}\right) \in\left\langle\alpha_{i} \widetilde{\alpha}_{i} F(2 k)_{n}\right\rangle^{N} \\
& \Leftrightarrow w F(2 k)_{n}\left\langle\alpha_{i} \widetilde{\alpha_{i}} F(2 k)_{n}\right\rangle^{N}=w^{\prime} F(2 k)_{n}\left\langle\alpha_{i} \widetilde{\alpha}_{i} F(2 k)_{n}\right\rangle^{N} .
\end{aligned}
$$

It follows that $\lambda$ is well-defined and injective. It is easy to see that it is also an onto homomorphism.

Lemma 2. Let $\beta$ be a $2 k$-string link with bottom meridians $y_{1}, y_{2}, \ldots, y_{k}$, $\widetilde{y_{k}}, \ldots, \widetilde{y_{2}}, \widetilde{y_{1}}$. There is an isomorphism

$$
\lambda^{\prime}: \frac{\pi(\beta)}{\left\langle y_{i} \widetilde{y}_{i}\right\rangle^{N} \pi(\beta)_{n}} \rightarrow \frac{\frac{\pi(\beta)}{\pi(\beta)_{n}}}{\left\langle y_{i} \widetilde{y_{i}} \pi(\beta)_{n}\right\rangle^{N}}
$$

defined by $\left(w\left\langle y_{i}{\widetilde{y_{i}}}\right\rangle^{N} \pi(\beta)_{n}\right) \lambda^{\prime}=w \pi(\beta)_{n}\left\langle y_{i} \widetilde{y_{i}} \pi(\beta)_{n}\right\rangle^{N}$, for any $w \in \pi(\beta)$.

Proof. It can be proved as Lemma 1.

Lemma 3. If $f: G \rightarrow H$ is a group epimorphism, then $f$ induces an isomorphism

$$
f^{\prime}: \frac{G}{\operatorname{ker}(f) G_{n}} \rightarrow \frac{H}{H_{n}}
$$


Proof. If $L \subseteq G$ is a subgroup, we have $((L) f) f^{-1}=\operatorname{ker}(f) L$. In particular, if $L=G_{n}$, we have $\left(\left(G_{n}\right) f\right) f^{-1}=\operatorname{ker}(f) G_{n}$, but $\left(G_{n}\right) f=H_{n}$, so $\left(H_{n}\right) f^{-1}=$ $\operatorname{ker}(f) G_{n}$. Therefore $f$ induces an isomorphism

$$
f^{\prime}: \frac{G}{\operatorname{ker}(f) G_{n}}=\frac{G}{\left(H_{n}\right) f^{-1}} \rightarrow \frac{H}{H_{n}} .
$$

Let $F(k)$ be the free group in $k$ generators $\alpha_{1}, \alpha_{2}, \ldots, \alpha_{k}$ and $F(2 k)$ be the free group in $2 k$ generators $\alpha_{1}, \alpha_{2}, \ldots, \alpha_{k}, \widetilde{\alpha_{k}}, \ldots, \widetilde{\alpha_{2}}, \widetilde{\alpha_{1}}$. We will denote by $\xi$ the epimorphism $\xi: F(2 k) \rightarrow F(k)$ given by $\left(\alpha_{i}\right) \xi=\alpha_{i}$ and $\left(\widetilde{\alpha}_{i}\right) \xi=\alpha_{i}^{-1}$ for any $i \in \underline{k}$. The kernel of $\xi$ is $\left\langle\alpha_{i} \tilde{\alpha}_{i}\right\rangle^{N}$, the normal subgroup of $F(2 k)$ generated by $\left\{\alpha_{i} \widetilde{\widetilde{\alpha}}_{i} \mid i \in \underline{k}\right\}$.

It follows from Lemma 3 that $\xi$ induces an isomorphism

$$
\xi^{\prime}: \frac{F(2 k)}{\left\langle\alpha_{i} \widetilde{\alpha}_{i}\right\rangle^{N} F(2 k)_{n}} \rightarrow \frac{F(k)}{F(k)_{n}} .
$$

Let ${\overline{S_{k}(1)_{n}}}_{n}=\left\{\beta \in S L(2 k) \mid \overline{\beta \cdot 1}_{n}\right.$ is the identity automorphism of $\left.\frac{F(k)}{F(k)_{n}}\right\}$. Clearly $\overline{S_{k}(1)_{n}}$ contains the stabilizer of 1 for Habegger-Lin's action $S_{k}(1)=$ $\{\beta \in S L(2 k) \mid \beta \cdot 1=1 \in S L(k)\}$.

THEOREM 4. Let $\beta \in S L(2 k)$. If $\beta \in{\overline{S_{k}(1)}}_{n}$ then there exists an epimorphism $\overline{\bar{\beta}}_{n}: \frac{F(k)}{F(k)_{n}} \rightarrow \frac{F(k)}{F(k)_{n}}$ such that the diagram

$$
\begin{array}{cccc}
\frac{F(2 k)}{F(2 k)_{n}} & & \bar{\beta}_{n} & \frac{F(2 k)}{F(2 k)_{n}} \\
\xi_{n} & & \downarrow_{n} \\
\frac{F(k)}{F(k)_{n}} & \stackrel{\bar{\beta}_{n}}{\longrightarrow} & \frac{F(k)}{F(k)_{n}}
\end{array}
$$

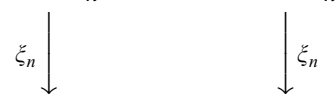

is commutative, where $\xi_{n}$ is induced from $\xi: F(2 k) \rightarrow F(k)$.

Proof. Let $\beta \in S L(2 k)$ have top meridians $x_{1}, x_{2}, \ldots, x_{k}, \widetilde{x_{k}}, \ldots, \widetilde{x_{2}}, \widetilde{x_{1}}$ and bottom meridians $y_{1}, y_{2}, \ldots, y_{k}, \widetilde{y_{k}}, \ldots, \widetilde{y_{2}}, \widetilde{y_{1}}$, and let $F(2 k)$ have generators $\alpha_{1}, \alpha_{2}, \ldots, \alpha_{k}, \widetilde{\alpha_{k}}, \ldots, \widetilde{\alpha_{2}}, \widetilde{\alpha_{1}}$ as we saw earlier. We have isomorphisms

$$
\frac{F(2 k)}{F(2 k)_{n}} \stackrel{\mu_{0}(\beta)_{n}}{\cong} \frac{\pi(\beta)}{\pi(\beta)_{n}} \stackrel{\left(\mu_{1}(\beta)_{n}\right)^{-1}}{\cong} \frac{F(2 k)}{F(2 k)_{n}}
$$

where $\left(\mu_{1}(\beta)_{n}\right)^{-1}$ sends $y_{i} \pi(\beta)_{n}$ to $\alpha_{i} F(2 k)_{n}$ and $\tilde{y}_{i} \pi(\beta)_{n}$ to $\tilde{\alpha}_{i} F(2 k)_{n}$, therefore it induces an isomorphism

$$
\left(\Gamma_{1}(\beta)_{n}\right)^{-1}: \frac{\frac{\pi(\beta)}{\pi(\beta)_{n}}}{\left\langle y_{i} \tilde{y}_{i} \pi(\beta)_{n}\right\rangle^{N}} \rightarrow \frac{\frac{F(2 k)}{F(2 k)_{n}}}{\left\langle\alpha_{i} \tilde{\alpha}_{i} F(2 k)_{n}\right\rangle^{N}},
$$


where $\left\langle u_{i}\right\rangle^{N}$ is the normal subgroup generated by $u_{i}, i \in \underline{k}$. By Lemma 2 there is an isomorphism between $\frac{\frac{\pi(\beta)}{\pi(\beta)_{n}}}{\left\langle y_{i} \tilde{y}_{i} \pi(\beta)_{n}\right\rangle^{N}}$ and $\frac{\pi(\beta)}{\left\langle y_{i} \tilde{y}_{i}\right\rangle^{N} \pi(\beta)_{n}}$. On the other side, $\beta \cdot 1$ is a $k$-string link and inclusion map induces an epimorphism $\sigma: \pi(\beta) \rightarrow$ $\pi(\beta \cdot 1)$ sending $y_{i}$ to $y_{i}$ and $\tilde{y}_{i}$ to $y_{i}^{-1}$, for any $i \in \underline{k}$.

By Lemma 3, $\sigma$ induces an isomorphism between

$$
\frac{\pi(\beta)}{\left\langle y_{i} \tilde{y}_{i}\right\rangle^{N} \pi(\beta)_{n}} \text { and } \frac{\pi(\beta \cdot 1)}{\pi(\beta \cdot 1)_{n}} .
$$

By Lemma 1, there is an isomorphism between

$$
\frac{\frac{F(2 k)}{F(2 k)_{n}}}{\left\langle\alpha_{i} \tilde{\alpha}_{i} F(2 k)_{n}\right\rangle^{N}} \text { and } \frac{F(2 k)}{\left\langle\alpha_{i} \tilde{\alpha}_{i}\right\rangle^{N} F(2 k)_{n}},
$$

and Lemma 3 applied to $\xi$ provides an isomorphism between

$$
\frac{F(2 k)}{\left\langle\alpha_{i} \tilde{\alpha}_{i}\right\rangle^{N} F(2 k)_{n}} \text { and } \frac{F(k)}{F(k)_{n}} .
$$

Putting all these data in a diagram, we have

(I)

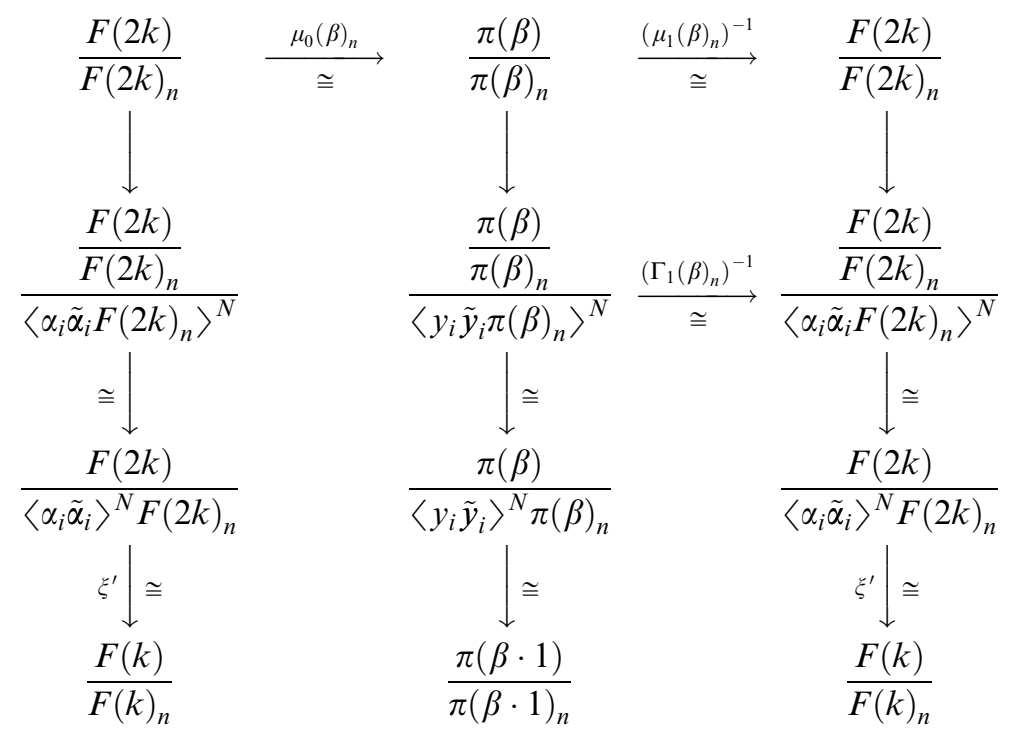

where $\cong$ represents isomorphisms and top vertical maps are quotient maps. 
Note that the $k$-string link $\beta \cdot 1$ has $x_{i}, i \in \underline{k}$, as top meridians and $\tilde{x}_{i}^{-1}$, $i \in \underline{k}$, as bottom meridians. Therefore $\left[\alpha_{i}\right] \mu_{0}(\beta \cdot 1)_{n}=\left[x_{i}\right]$, where [ ] denotes the equivalence classes in the quotient groups $\frac{F(n)}{F(k)_{n}}$ and $\frac{\pi(\beta \cdot 1)}{\pi(\beta \cdot 1)_{n}}$. Similarly,
$\left[\alpha_{i}\right] \mu_{1}(\beta \cdot 1)_{n}=\left[\tilde{x}_{i}^{-1}\right]$. Then

$$
\left[\alpha_{i}\right] \mu_{0}(\beta \cdot 1)_{n} \mu_{1}(\beta \cdot 1)_{n}^{-1}=\left[x_{i}\right] \mu_{1}(\beta \cdot 1)_{n}^{-1} .
$$

Thus we have the equivalence

$$
\begin{aligned}
{\left[x_{i}\right] \mu_{1}(\beta \cdot 1)_{n}^{-1} } & =\left[\tilde{x}_{i}^{-1}\right] \mu_{1}(\beta \cdot 1)_{n}^{-1} \\
& \Leftrightarrow\left[\alpha_{i}\right] \mu_{0}(\beta \cdot 1)_{n} \mu_{1}(\beta \cdot 1)_{n}^{-1}=\left[\alpha_{i}\right] \\
& \Leftrightarrow\left[\alpha_{i}\right](\overline{\beta \cdot 1})_{n}=\left[\alpha_{i}\right] .
\end{aligned}
$$

Suppose now that $\beta \in{\overline{S_{k}(1)_{n}}}_{\text {. Then }} \overline{\beta \cdot 1}_{n}$ is the identity map. Then $\left[x_{i}\right] \mu_{1}(\beta \cdot 1)_{n}^{-1}=\left[\tilde{x}_{i}^{-1}\right] \mu_{1}(\beta \cdot 1)_{n}^{-1}$, but $\mu_{1}(\beta \cdot 1)_{n}^{-1}$ is an isomorphism, so $\left[x_{i}\right]=$ $\left[\tilde{x}_{i}^{-1}\right]$ in $\frac{\pi(\beta \cdot 1)}{\pi(\beta \cdot 1)_{n}}$, and we have seen that $\frac{\pi(\beta \cdot 1)}{\pi(\beta \cdot 1)_{n}}$ is isomorphic to

$$
\frac{\frac{\pi(\beta)}{\pi(\beta)_{n}}}{\left\langle y_{i} \tilde{y}_{i} \pi(\beta)_{n}\right\rangle^{N}} .
$$

It follows that, in this last group, the class of $x_{i}$ is equal to the class of $\tilde{x}_{i}^{-1}$. Now observe that $\mu_{0}(\beta)_{n}: \frac{F(2 k)}{F(2 k)_{n}} \rightarrow \frac{\pi(\beta)}{\pi(\beta)_{n}}$ sends $\alpha_{i} \tilde{\alpha}_{i} F(2 k)_{n}$ to $x_{i} \tilde{x}_{i} \pi(\beta)_{n}$, for any $i \in \underline{k}$. Therefore $\mu_{0}(\beta)_{n}$ induces a homomorphism

$$
\Gamma_{0}(\beta)_{n}: \frac{\frac{F(2 k)}{F(2 k)_{n}}}{\left\langle\alpha_{i} \tilde{\alpha}_{i} F(2 k)_{n}\right\rangle^{N}} \rightarrow \frac{\frac{\pi(\beta)}{\pi(\beta)_{n}}}{\left\langle y_{i} \tilde{y}_{i} \pi(\beta)_{n}\right\rangle^{N}} .
$$

We have thus a commutative diagram

(II)

$$
\begin{aligned}
& \begin{array}{ccccc}
\frac{F(2 k)}{F(2 k)_{n}} & \stackrel{\mu_{0}(\beta)_{n}}{\cong} & \frac{\pi(\beta)}{\pi(\beta)_{n}} & \stackrel{\left(\mu_{1}(\beta)_{n}\right)^{-1}}{\cong} & \frac{F(2 k)}{F(2 k)_{n}} \\
\downarrow & \downarrow & &
\end{array} \\
& \frac{\frac{F(2 k)}{F(2 k)_{n}}}{\left\langle\alpha_{i} \tilde{\alpha}_{i} F(2 k)_{n}\right\rangle^{N}} \stackrel{\Gamma_{0}(\beta)_{n}}{\longrightarrow} \frac{\frac{\pi(\beta)}{\pi(\beta)_{n}}}{\left\langle y_{i} \tilde{y}_{i} \pi(\beta)_{n}\right\rangle^{N}} \stackrel{\left(\Gamma_{1}(\beta)_{n}\right)^{-1}}{\longrightarrow} \frac{\frac{F(2 k)}{F(2 k)_{n}}}{\left\langle\alpha_{i} \tilde{\alpha}_{i} F(2 k)_{n}\right\rangle^{N}}
\end{aligned}
$$

where the vertical maps are quotient maps. 
Diagrams (I) and (II) provide a commutative diagram

(III)

$$
\begin{array}{ccccc}
\frac{F(2 k)}{F(2 k)_{n}} & \stackrel{\mu_{0}(\beta)_{n}}{\longrightarrow} \frac{\pi(\beta)}{\pi(\beta)_{n}} & \stackrel{\left(\mu_{1}(\beta)_{n}\right)^{-1}}{\longrightarrow} & \frac{F(2 k)}{F(2 k)_{n}} \\
\xi_{n} & \downarrow & & \downarrow \xi_{n} \\
\frac{F(k)}{F(k)_{n}} & \stackrel{\Gamma_{0}^{\prime}(\beta)_{n}}{\longrightarrow} \frac{\pi(\beta \cdot 1)}{\pi(\beta \cdot 1)_{n}} \stackrel{\left(\Gamma_{1}^{\prime}(\beta)_{n}\right)^{-1}}{\longrightarrow} \frac{F(k)}{F(k)_{n}}
\end{array}
$$

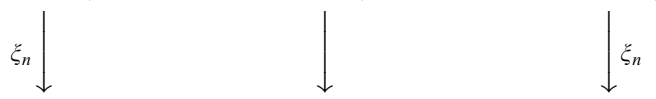

where $\xi_{n}$ is induced from $\xi: F(2 k) \rightarrow F(k)$.

Let us recall that $\mu_{0}(\beta)_{n}\left(\mu_{1}(\beta)_{n}\right)^{-1}=\bar{\beta}_{n}$ and denote $\Gamma_{0}^{\prime}(\beta)_{n}\left(\Gamma_{1}^{\prime}(\beta)_{n}\right)^{-1}$ by $\overline{\bar{\beta}}_{n}$. Therefore we have a commutative diagram

$$
\begin{array}{cccc}
\frac{F(2 k)}{F(2 k)_{n}} & \stackrel{\bar{\beta}_{n}}{\longrightarrow} \frac{F(2 k)}{F(2 k)_{n}} \\
\xi_{n} & & \xi_{n} \\
\frac{F(k)}{F(k)_{n}} & \stackrel{\bar{\beta}_{n}}{\longrightarrow} \frac{F(k)}{F(k)_{n}}
\end{array}
$$

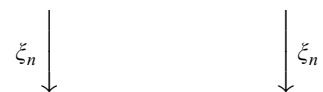

Let $\overline{S_{k}(1)}=\{\beta \in S L(2 k) \mid \widetilde{\beta \cdot 1}$ is the identity automorphism of $\widetilde{F(k)}\}$. Then $S_{k}(1) \subseteq \overline{S_{k}(1)}=\bigcap_{n} \overline{S_{k}(1)}{ }_{n}$.

COROLlary 5. If $\beta \in \overline{S_{k}(1)}$, then there exists an epimorphism $\tilde{\tilde{\beta}}: \widetilde{F(k)} \rightarrow$ $\widetilde{F(k)}$ such that the diagram

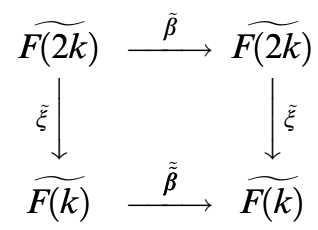

is commutative, where $\tilde{\xi}$ is induced from $\xi$.

If $\beta \in S L(2 k)$ and $f \in S L(k)$ we have also a previously defined HabeggerLin's action $f \cdot \beta$. Thus we can consider ${ }_{k} \overline{S(1)}_{n}=\left\{\beta \in S L(2 k) \mid \overline{1 \cdot \beta}_{n}\right.$ is the identity automorphism of $\left.\frac{F(k)}{F(k)_{n}}\right\}$. Then ${ }_{k} \overline{S(1)}_{n}$ contains the stabilizer of 1 for Habegger-Lin's action ${ }_{k} S(1)=\{\beta \in S L(2 k) \mid 1 \cdot \beta=1 \in S L(k)\}$. Similarly we can define ${ }_{k} \overline{S(1)}=\{\beta \in S L(2 k) \mid \widetilde{1 \cdot \beta}$ is the identity automorphism of $\widetilde{F(k)}\}=$ $\bigcap_{n k} \overline{S(1)}_{n}$. 
THEOREM 6. Let $\beta \in S L(2 k) . \quad \beta \in{\overline{S_{k}(1)}}_{n} \cap_{k} \overline{S(1)}_{n}$ if and only if there exists an automorphism $\overline{\bar{\beta}}_{n}: \frac{F(k)}{F(k)_{n}} \rightarrow \frac{F(k)}{F(k)_{n}}$ such that diagram $\left(^{*}\right)$ is commutative.

Proof. If $\beta \in{\overline{S_{k}(1)_{n}}}_{n} \overline{S(1)}_{n}$, then $\beta \in{\overline{S_{k}(1)}}_{n}$ and $\beta^{R} \in{\overline{S_{k}(1)}}_{n}$, so we have a commutative diagram

$$
\begin{array}{ccccc}
\frac{F(2 k)}{F(2 k)_{n}} & \stackrel{\bar{\beta}_{n}}{\longrightarrow} \frac{F(2 k)}{F(2 k)_{n}} \stackrel{\bar{\beta}_{n}^{-1}=\overline{\beta_{n}^{R}}}{\longrightarrow} \frac{F(2 k)}{F(2 k)_{n}} \\
\xi_{n} & & \xi_{n} & & \downarrow \xi_{n} \\
\frac{F(k)}{F(k)_{n}} & \stackrel{\bar{\beta}_{n}}{\longrightarrow} \frac{F(k)}{F(k)_{n}} \stackrel{\overline{\overline{\beta_{n}^{R}}}}{\longrightarrow} \frac{F(k)}{F(k)_{n}} .
\end{array}
$$

It follows that $\overline{\bar{\beta}}_{n} \overline{\overline{\beta_{n}^{R}}}$ is the identity map. Similarly $\overline{\overline{\beta_{n}^{R}}} \overline{\bar{\beta}}_{n}$ is the identity map.

Conversely suppose we have a commutative diagram $\left(^{*}\right)$ with $\overline{\overline{\beta_{n}}}$ an

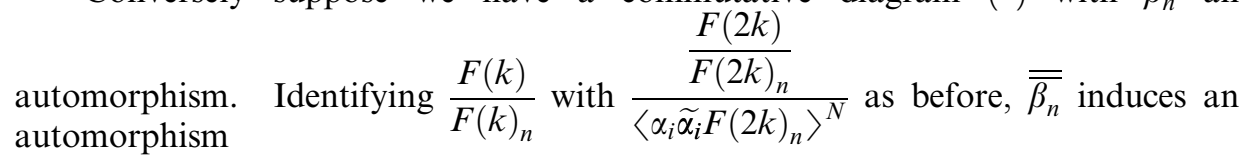

$$
\overline{\bar{\beta}}_{n}^{\prime}: \frac{\frac{F(2 k)}{F(2 k)_{n}}}{\left\langle\alpha_{i} \widetilde{\alpha}_{i} F(2 k)_{n}\right\rangle^{N}} \rightarrow \frac{\frac{F(2 k)}{F(2 k)_{n}}}{\left\langle\alpha_{i} \widetilde{\alpha}_{i} F(2 k)_{n}\right\rangle^{N}}
$$

and we have a commutative diagram

$$
\begin{array}{ccccc}
\frac{F(2 k)}{F(2 k)_{n}} & \stackrel{\mu_{0}(\beta)_{n}}{\longrightarrow} & \frac{\pi(\beta)}{\pi(\beta)_{n}} & \stackrel{\left(\mu_{1}(\beta)_{n}\right)^{-1}}{\longrightarrow} & \frac{F(2 k)}{F(2 k)_{n}} \\
\frac{F(2 k)}{F(2 k)_{n}} & \stackrel{\bar{\beta}_{n}^{\prime} \Gamma_{1}(\beta)_{n}}{\longrightarrow} \frac{\frac{\pi(\beta)}{\pi(\beta)_{n}}}{\left.\left\langle\alpha_{i} \tilde{\alpha}_{i} F(2 k)_{n}\right\rangle^{N} \pi(\beta)_{n}\right\rangle^{N}} \stackrel{\left(\Gamma_{1}(\beta)_{n}\right)^{-1}}{\longrightarrow} \frac{\frac{F(2 k)}{F(2 k)_{n}}}{\left\langle\alpha_{i} \tilde{\alpha}_{i} F(2 k)_{n}\right\rangle^{N}}
\end{array}
$$

where the horizontal maps are isomorphisms and the vertical maps are quotient maps. Since $\mu_{0}(\beta)_{n}$ sends $\alpha_{i} \widetilde{\alpha}_{i} F(2 k)_{n}$ to $x_{i} \widetilde{x}_{i} \pi(\beta)_{n}$, it follows that, in

$$
\frac{\frac{\pi(\beta)}{\pi(\beta)_{n}}}{\left\langle y_{i} \widetilde{y}_{i} \pi(\beta)_{n}\right\rangle^{N}} \cong \frac{\pi(\beta \cdot 1)}{\pi(\beta \cdot 1)_{n}}
$$

the class of $x_{i}$ is equals to the class of $\widetilde{x}_{i}^{-1}$. But the classes of $x_{i}, i \in \underline{k}$, are the top meridians of $\beta \cdot 1$ and the classes of $\widetilde{x}_{i}^{-1}, i \in \underline{k}$, are the bottom meridians of $\beta \cdot 1$, so $\overline{\beta \cdot 1}_{n}=\mu_{0}(\beta \cdot 1)_{n}\left(\mu_{1}(\beta \cdot 1)_{n}\right)^{-1}$ is the identity map. Therefore 
$\beta \in{\overline{S_{k}(1)}}_{n}$. The same argument with $\beta$ replaced by $\beta^{R}$ and $\overline{\bar{\beta}}_{n}$ replaced by $\overline{\bar{\beta}}_{n}^{-1}$ shows that $\beta^{R} \in{\overline{S_{k}(1)}}_{n}$.

COROLlaRY 7. $\beta \in \overline{S_{k}(1)} \cap_{k} \overline{S(1)}$ if and only if there exists an automorphism $\tilde{\tilde{\beta}}: \widetilde{F(k)} \rightarrow \widetilde{F(k)}$ such that diagram $\left.{ }^{* *}\right)$ is commutative.

Proof. If $\beta \in \overline{S_{k}(1)}$ and $\beta^{R} \in \overline{S_{k}(1)}$, then they are in all $\overline{S_{k}(1)}$ and we have automorphisms $\overline{\overline{\beta_{n}}}$ that together provide automorphism $\tilde{\tilde{\beta}}$.

Conversely suppose we have automorphism $\tilde{\tilde{\beta}}$ making diagram (**) commutative.

The natural map $\frac{F}{F_{n}} \rightarrow \frac{\tilde{F}}{\tilde{F}_{n}}$, where $F=F(k)$ or $F(2 k)$, is an isomorphism (see [9]). Therefore we have commutative diagrams $(*)$ for every $n$, where $\overline{\bar{\beta}}_{n}$ is an automorphism. Then $\beta, \beta^{R} \in \bar{S}_{k}(1)_{n}$, for every $n$. Thus $\beta, \beta^{R} \in \overline{S_{k}(1)}$.

THEOREM 8. The intersection ${\overline{S_{k}(1)}}_{n} \cap_{k} \overline{S(1)}$, is closed in relation to the multiplication.

Proof. By Theorem 6, $\beta \in{\overline{S_{k}(1)}}_{n} \cap_{k} \overline{S(1)}_{n}$ if and only if there exists automorphism $\overline{\bar{\beta}}_{n}$ commuting diagram $\left(^{*}\right)$. Thus if $\beta, \gamma \in{\overline{S_{k}(1)}}_{n} \cap_{k} \overline{S(1)}_{n}$, then there are automorphisms $\overline{\bar{\beta}}_{n}$ and $\overline{\bar{\gamma}}_{n}$ making diagrams below commutatives.

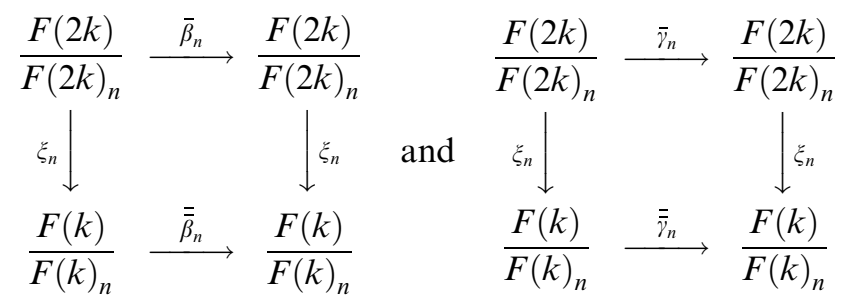

Therefore the automorphism $\overline{\bar{\beta}}_{n} \overline{\bar{\gamma}}_{n}$ makes the diagram

$$
\begin{array}{ccc}
\frac{F(2 k)}{F(2 k)_{n}} & \stackrel{\overline{(\beta \gamma)}_{n}}{\longrightarrow} \frac{F(2 k)}{F(2 k)_{n}} \\
\xi_{n} & & \downarrow \xi_{n} \\
\frac{F(k)}{F(k)_{n}} & \stackrel{\overline{\bar{\beta}}_{n} \overline{\bar{\gamma}}_{n}}{\longrightarrow} \frac{F(k)}{F(k)_{n}}
\end{array}
$$

commutative. Therefore, by Theorem $6, \beta \gamma \in{\overline{S_{k}(1)}}_{n} \cap_{k} \overline{S(1)}{ }_{n}$.

Similarly, using Corollary 7, we see that

COROLlaRY 9. $\overline{S_{k}(1)} \cap_{k} \overline{S(1)}$ is closed in relation to the multiplication. 


\section{Concordance}

Definition 4. $k$-string links $f, g: \underline{k} \times I \rightarrow D \times I$ are said to be concordant if there is an embedding $F: \underline{k} \times I \times \bar{I} \rightarrow D \times I \times I$ such that $\left.F\right|_{k \times I \times\{0\}}=f$, $\left.F\right|_{\underline{k} \times I \times\{1\}}=g$ and $\left.F\right|_{\underline{k} \times \partial I \times I}=\left(\left.j_{0}\right|_{\underline{k} \times \partial I}\right) \times i d_{I}$ where $i d_{I}: I \rightarrow I$ is the identity map.

The set of concordance classes of $k$-string links with the operation induced by the multiplication of $k$-string links is a group where the inverse of the class of $f$ is the class of $f^{R}$ (see [4]). This group will be denoted by $\operatorname{CSL}(k)$.

Habegger-Lin's actions induce actions $\operatorname{CSL}(2 k) \times C S L(k) \rightarrow C S L(k)$ and $C S L(k) \times C S L(2 k) \rightarrow C S L(k)$ (see [6]). The stabilizer of 1 for both of these actions is the same (see [6]) and will be denoted by $S_{k}^{C}(1)=\{\beta \in C S L(2 k) \mid \beta \cdot 1=$ $1 \in \operatorname{CSL}(k)\}=\{\beta \in \operatorname{CSL}(2 k) \mid 1 \cdot \beta=1 \in \operatorname{CSL}(k)\}$.

Definition 5. Consider $k$-component links $L_{0}$ and $L_{1}$. A (link) concordance between $L_{0}$ and $L_{1}$ is an embedding:

$$
H:\left(\bigsqcup_{i=1}^{k} S^{1}\right) \times I \rightarrow S^{3} \times I
$$

such that $H(x, 0)=\left(L_{0}(x), 0\right)$ and $H(x, 1)=\left(L_{1}(x), 1\right)$.

If $k$-string links $f$ and $g$ are concordant then their closures $\hat{f}$ and $\hat{g}$ are (link) concordant. Actually, we have

THEOREM 10 (Habegger-Lin). Suppose $f, g \in C S L(k)$, then $\hat{f}=\hat{g}$ (that is, the closures of their representatives are link concordant) if and only if there exists $\beta \in S_{k}^{C}(1)$ such that $\beta \cdot f=g$.

Proof. See [6].

If $f$ and $g$ are concordant $k$-string links, then $\bar{f}_{n}=A_{n}(f)=A_{n}(g)=\bar{g}_{n}$ (see [6]). Considering the nilpotent completion we also have $\tilde{f}=A(f)=A(g)=\tilde{g}$. Furthermore the $A_{n}(f)$ are braid-like automorphisms of $\frac{F(k)}{F(k)_{n}}$, that is (i) they send the class of each generator $\alpha_{i}$ into a conjugate of itself and (ii) they send the class of the product $\alpha_{1} \alpha_{2} \cdots \alpha_{n}$ into itself. Thus, if we denote the group of braid-like automorphisms of $\frac{F(k)}{F(k)_{n}}$ by $A u t_{0}\left(\frac{F(k)}{F(k)_{n}}\right)$, we have a homomorphism $A_{n}: C S L(k) \rightarrow A_{0}\left(\frac{F(k)}{F(k)_{n}}\right) \begin{aligned} & F(k)_{n} \\ & \text { called Artin representation. This homomorphism }\end{aligned}$ is actually an epimorphism (see [6]).

An automorphism that satisfies (i) above is called special.

Let $\overline{S_{k}^{C}(1)_{n}}=\left\{\beta \in C S L(2 k) \mid \overline{\beta \cdot 1}_{n}\right.$ is the identity automorphism of $\left.\frac{F(k)}{F(k)_{n}}\right\}$ $=\left\{\beta \in C S L(2 k) \mid \beta \cdot 1 \in \operatorname{ker} A_{n}\right\},{ }_{k} \overline{S^{C}(1)_{n}}=\left\{\beta \in C S L(2 k) \mid 1 \cdot \beta \in \operatorname{ker} A_{n}\right\}, \overline{S_{k}^{C}(1)}$ 


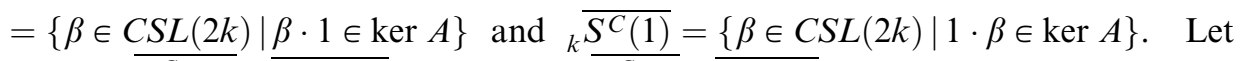
$S_{n}(1)=\overline{S_{k}^{C}(1)_{n}} \cap \overline{S^{C}(1)_{n}}$ and $S(1)=\overline{S_{k}^{C}(1)} \cap_{k} \overline{S^{C}(1)}$.

THEOREM 11. $S_{n}(1)$ and $S(1)$ are groups.

Proof. From Theorem 8 and Corollary 9 they are closed for multiplication.

On the other side, if $\beta \in \bar{S}_{k}^{C}(1)_{n}$, then $\overline{\beta \cdot 1}_{n}=i d$, the identity map. Then $i d=\left(\overline{\beta \cdot 1}_{n}\right)^{-1}=\overline{(\beta \cdot 1)^{-1}}=\left(\overline{1 \cdot \beta^{-1}}\right)_{n}$, so $\beta^{-1} \in \overline{S^{C}(1)_{n}}$. Therefore $S_{n}(1)$ is a group. Similarly $S(1)$ is a group. actions.

Clearly $S_{n}(1)$ and $S(1)$ contain $S_{k}^{C}(1)$, the stabilizer of 1 for Habegger-Lin's

Theorem 6 and Corollary 7 provide the following results:

THEOREM 12. Let $\beta \in \operatorname{CSL}(2 k) . \quad \beta \in S_{n}(1)$ if and only if there is a special automorphism $\overline{\bar{\beta}}_{n}: \frac{F(k)}{F(k)_{n}} \rightarrow \frac{F(k)}{F(k)_{n}}$ such that diagram (*) is commutative.

COROLlaRY 13. $\beta \in S(1)$ if and only if there is a special automorphism $\tilde{\tilde{\beta}}: \widetilde{F(k)} \rightarrow \widetilde{F(k)}$ such that diagram $\left.{ }^{* *}\right)$ is commutative.

In particular, if $\beta \in S_{k}^{C}(1)$, the stabilizer of 1 for Habegger-Lin's action, we have special automorphisms $\overline{\bar{\beta}}_{n}$ and $\tilde{\tilde{\beta}}$ as above.

Let $g \times 1$ represent the $2 k$-string link obtained from a $k$-string link $g$ by adding $k$ straight strings at its end (see Fig. 4).

LEMma 14. Let $f, g \in \operatorname{CSL}(k)$ and $\gamma \in \operatorname{CSL}(2 k) . \gamma \cdot f=g$ if and only if $\gamma$ is of the form $g \times 1 \beta f^{-1} \times 1$, where $\beta \in S_{k}^{C}(1)$.

Proof. See [1]-Lemma 4.

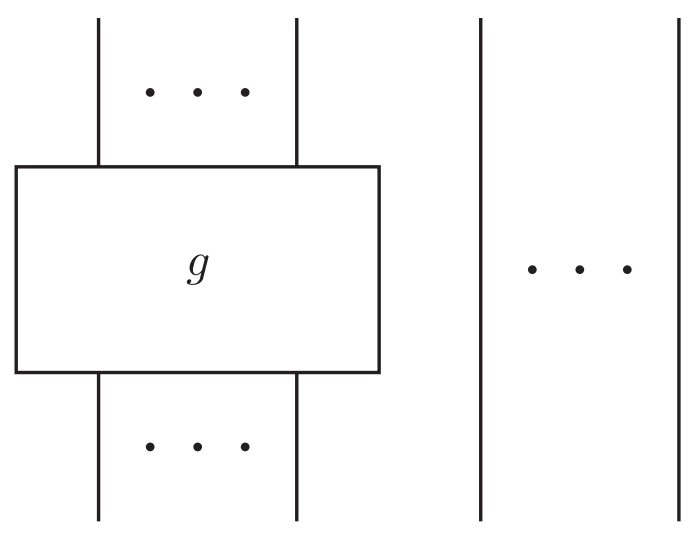

FIGURE 4. The string link $g \times 1$. 
THEOREM 15. If the closures of $k$-string links $f$ and $g$ are link concordant then, for every $n$, there exists a commutative diagram

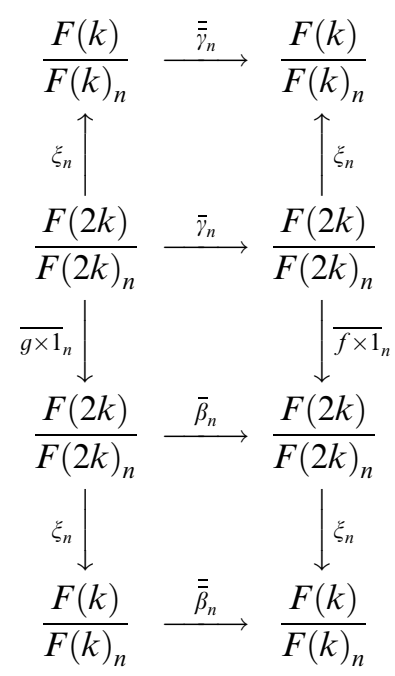

where $\bar{\beta}_{n}$ and $\bar{\gamma}_{n}$ are braid-like automorphisms and $\overline{\bar{\beta}}_{n}, \overline{\bar{\gamma}}_{n}$ are special automorphisms.

Proof. If $\hat{f}$ and $\hat{g}$ are link concordant, then, by Habegger-Lin's Theorem (Theorem 10), there exists $\gamma \in S_{k}^{C}(1)$ such that $\gamma \cdot f=g$ (here we are using $f$ and $g$ to represent also the concordance classes of $f$ and $g$ ).

By Lemma 14, $\gamma=g \times 1 \beta f^{-1} \times 1$, where $\beta \in S_{k}^{C}(1)$.

By Theorem 12, we have a commutative diagram as stated.

COROLlaRY 16. If the closures of $k$-string links $f$ and $g$ are link concordant, then there is a commutative diagram

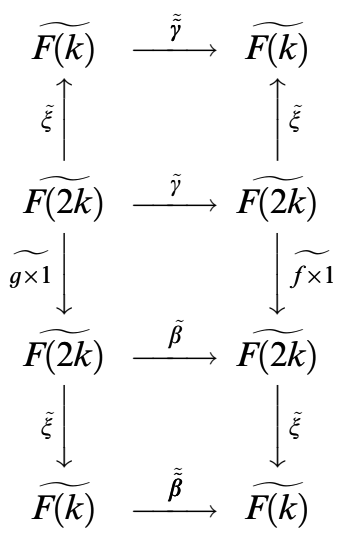

where $\tilde{\beta}, \tilde{\gamma}$ are braid-like automorphisms and $\tilde{\tilde{\beta}}, \tilde{\tilde{\gamma}}$ are special automorphisms. 
Let $K_{n}=\operatorname{ker} \xi_{n}$, that is $K_{n}=\left\langle\alpha_{i} \widetilde{\alpha}_{i} F(2 k)_{n} \mid i \in \underline{k}\right\rangle^{N}$, the normal subgroup of $\frac{F(2 k)}{F(2 k)_{n}}$ generated by $\alpha_{i} \widetilde{\alpha}_{i} F(2 k)_{n}, i \in \underline{k}$, and let $K=\operatorname{ker} \tilde{\xi}$.

Proposition 17. If the closures of $k$-string links $f$ and $g$ are concordant, then there is $\beta \in S_{k}^{C}(1)$ s.t., for all $n \geq 2,\left(K_{n}\right) \overline{\beta_{n}} \subseteq K_{n}$ and $\left(\left(K_{n}\right) \overline{g \times 1_{n}}\right) \overline{\beta_{n}} \subseteq$ $\left(K_{n}\right) \overline{f \times 1}_{n}$.

Proof. According to the proof of Theorem 15, if $\hat{f}$ and $\hat{g}$ are concordant, then there exists $\beta \in S_{k}^{C}(1)$ such that $\gamma=g \times 1 \beta f^{-1} \times 1 \in S_{k}^{C}(1)$. By Theorem 12 and the fact that $S_{k}^{C}(1) \subseteq S_{n}(1)$, we see that $\beta \in S_{k}^{C}(1)$ implies $\left(K_{n}\right) \bar{\beta}_{n} \subseteq K_{n}$ and $\gamma \in S_{k}^{C}(1) \quad$ implies $\left(K_{n}\right) \bar{\gamma}_{n} \subseteq K_{n}$, so $\left(K_{n}\right) \overline{g \times 1}_{n} \bar{\beta}_{n} \bar{f}^{-1} \times 1_{n} \subseteq K_{n}$. Then $\left(\left(K_{n}\right) \overline{g \times 1}_{n}\right) \bar{\beta}_{n} \subseteq\left(K_{n}\right) \overline{f \times 1}_{n}$.

COROLlaRY 18. If the closures of $k$-string links $f$ and $g$ are concordant, then there is $\beta \in S_{k}^{C}(1)$ s.t. $\left(\lim _{n} K_{n}\right) \tilde{\beta} \subseteq \lim _{n} K_{n}$ and $\left(\left(\lim _{n} K_{n}\right) g \times 1\right) \tilde{\beta} \subseteq$ $\left(\lim _{n} K_{n}\right) \widetilde{f \times 1}$.

COROLlaRY 19. If the closures of $k$-string links $f$ and $g$ are concordant, then there is $\beta \in S_{k}^{C}(1)$ s.t. $\left(K_{n}\right) \bar{\beta}_{n}=K_{n}$ and $\left(\left(K_{n}\right) \overline{g \times 1}_{n}\right) \bar{\beta}_{n}=\left(K_{n}\right) \overline{f \times 1}_{n}$.

Proof. It follows from Proposition 17 using $\beta^{-1}$.

COROLlaRY 20. If the closures of $k$-string links $f$ and $g$ are concordant, then there is $\beta \in S_{k}^{C}(1)$ s.t. $\left(\lim _{\longleftarrow} K_{n}\right) \tilde{\beta}=\lim _{\longleftarrow} K_{n}$ and $\left(\left(\lim _{\longleftarrow} K_{n}\right) \widetilde{g \times 1}\right) \tilde{\beta}=\left(\lim _{\leftarrow} K_{n}\right) \widetilde{f \times 1}$.

Definition 6. If $L$ is a link, the fundamental group of the complement of $L$ is called the group of $L$ and is denoted by $G(L)$.

By [12], the natural homomorphism $p_{f}: \pi(f) \rightarrow G(\hat{f})$ is onto with kernel normally generated by the commutators $\left[x_{i}(f), \lambda_{i}(f)\right]$, for any $i \in \underline{k}$, where $x_{i}(f)$ are the top meridians of $f$ and $\lambda_{i}(f)$ are correspondent longitudes of $f$. Thus we have, for every $n \geqslant 2$, an induced epimorphism $\left(p_{f}\right)_{n}: \frac{\pi(f)}{\pi(f)_{n}} \rightarrow \frac{G(\hat{f})}{G(\hat{f})_{n}}$. Let $\left(q_{f}\right)_{n}=\left(\mu_{0}(f)_{n}\right)\left(p_{f}\right)_{n}: \frac{F(k)}{F(k)_{n}} \rightarrow \frac{G(\hat{f})}{G(\hat{f})_{n}}$. Then $\left(q_{f}\right)_{n}$ is an epimorphism that sends $\alpha_{i} F(k)_{n}$ to $u_{i} G(\hat{f})_{n}$, where $u_{i} \in G(\hat{f})$ is a meridian for the link $\hat{f}$ arising from $x_{i}(f)$.

Considering the commutative diagram

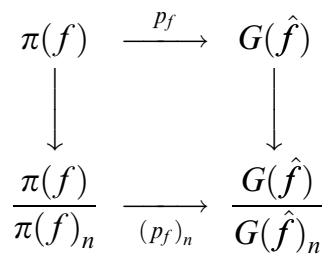


where the vertical maps are quocient maps, and the fact that $p_{f}$ is onto, we see that $\operatorname{ker}\left(\left(p_{f}\right)_{n}\right)=\frac{\left(G(\hat{f})_{n}\right)\left(p_{f}\right)^{-1}}{\pi(f)_{n}}=\frac{\left(\left(\pi(f)_{n}\right) p_{f}\right)\left(p_{f}\right)^{-1}}{\pi(f)_{n}}=\frac{\left(\operatorname{ker} p_{f}\right) \pi(f)_{n}}{\pi(f)_{n}}$ is the normal subgroup of $\frac{\pi(f)}{\pi(f)_{n}}$ generated by the commutators of the form $\left[x_{i}(f) \pi(f)_{n}, \lambda_{i}(f) \pi(f)_{n}\right]$, for $i \in \underline{k}$.

Since $\bar{f}_{n}$ conjugates the classes of the meridians $\alpha_{i} F(k)_{n}$ by the classes of the correspondent longitudes (see [6]), and remembering that $\mu_{0}(f)_{n}$ is an isomorphism, we have that $\operatorname{ker}\left(q_{f}\right)_{n}=\left\langle\left(\alpha_{i} F(k)_{n}\right) \bar{f}_{n} \alpha_{i}^{-1} F(k)_{n} \mid i \in \underline{k}\right\rangle^{N}$. It follows that $\operatorname{ker}\left(\xi_{n}\left(q_{f}\right)_{n}\right)=\left\langle\left(\alpha_{i} F(2 k)_{n}\right) \overline{f \times 1_{n}} \alpha_{i}^{-1} F(2 k)_{n}, \alpha_{i} \widetilde{\alpha}_{i} F(2 k)_{n} \mid i \in \underline{k}\right\rangle^{N}$.

Let $f$ be a $k$-string link. For each $n \geqslant 2$, consider the following diagram:

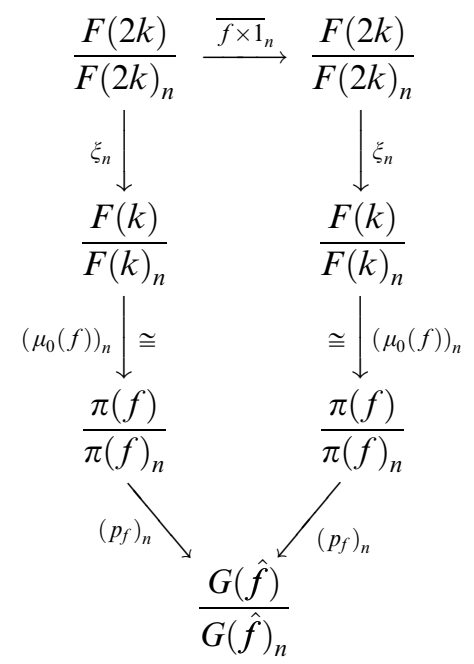

It follows from [12]-Proposition 1 that the above diagram is commutative. We have already denoted $\left(\mu_{0}(f)\right)_{n}\left(p_{f}\right)_{n}$ by $\left(q_{f}\right)_{n}$.

Definition 7. Given a $k$-string link $f$, the $n$-level group diagram for $f$, $n \geqslant 2$, is the commutative diagram:

$$
\begin{array}{ccc}
\frac{F(2 k)}{F(2 k)_{n}} & \stackrel{\overline{f \times 1}_{n}}{\longrightarrow} \frac{F(2 k)}{F(2 k)_{n}} \\
\frac{F(k)}{F(k)_{n}} & & \frac{F(k)}{F(k)_{n}} \\
\xi_{n} & & \xi_{\left(q_{f}\right)_{n}} \\
& & \frac{G(\hat{f})}{G(\hat{f})_{n}}
\end{array}
$$


Given $n$-level group diagrams for all $n$ and taking inverse limits, we have the group diagram for $f$ :

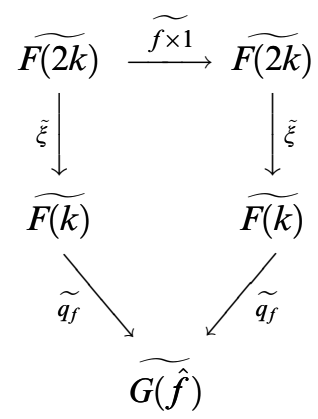

Definition 8. Given $n$-level group diagrams for $k$-string links $f$ and $g$, an $n$-level braid-special isomorphism between them is a commutative diagram:

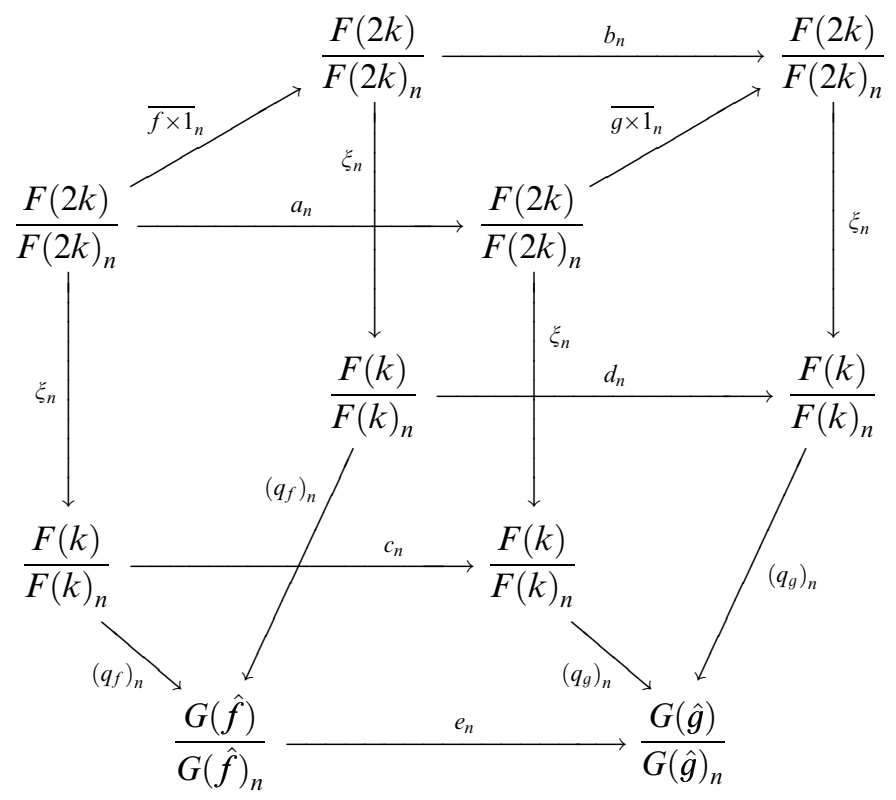

where $a_{n}, b_{n}$ are braid-like isomorphisms and $c_{n}, d_{n}, e_{n}$ are special isomorphisms.

DeFINITION 9. Given group diagrams for $k$-string links $f$ and $g$, a braidspecial isomorphism between them is a commutative diagram: 


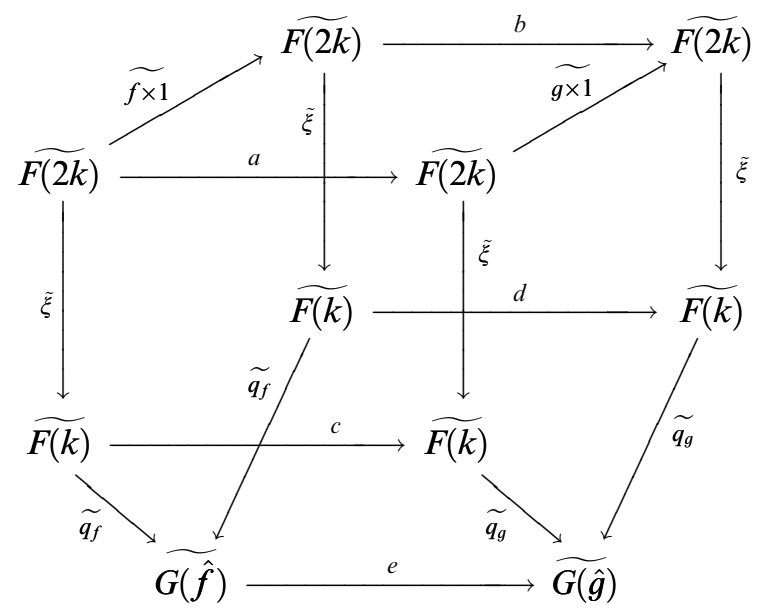

where $a, b$ are braid-like isomorphisms and $c, d, e$ are special isomorphisms.

THEOREM 21. Let $L_{1}, L_{2}$ be k-links and let $f, g$ be $k$-string links with $\hat{f}=L_{1}$ and $\hat{g}=L_{2}$. If $L_{1}$ and $L_{2}$ are concordant, then, for each $n \geqslant 2$, there exists an $n$-level braid-special isomorphism between the n-level group diagram of $f$ and the $n$-level group diagram of $g$.

Proof. Since $\hat{f}$ and $\hat{g}$ are concordant, by Theorem 15, there exists, for each $n \geqslant 2$, a commutative diagram

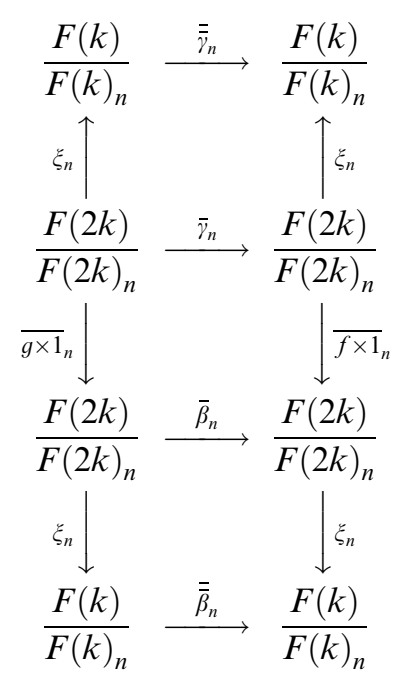

where $\bar{\beta}_{n}$ and $\bar{\gamma}_{n}$ are braid-like automorphisms and $\overline{\bar{\beta}}_{n}, \overline{\bar{\gamma}}_{n}$ are special automorphisms. 
It follows from Corollary 19 and the fact that, as we saw,

$$
\begin{aligned}
\operatorname{ker}\left(\xi_{n}\left(q_{f}\right)_{n}\right) & =\left\langle\left(\alpha_{i} F(2 k)_{n}\right) \overline{f \times 1_{n}} \alpha_{i}^{-1} F(2 k)_{n}, \alpha_{i} \tilde{\alpha}_{i} F(2 k)_{n} \mid i \in \underline{k}\right\rangle^{N} \\
& =\left\langle\left(\alpha_{i} F(2 k)_{n}\right) \overline{f \times 1}_{n} \tilde{\alpha}_{i} F(2 k)_{n}, \alpha_{i} \tilde{\alpha}_{i} F(2 k)_{n} \mid i \in \underline{k}\right\rangle^{N} \\
& =\left\langle\left(\alpha_{i} F(2 k)_{n}\right) \overline{f \times 1}_{n}\left(\tilde{\alpha}_{i} F(2 k)_{n}\right) \overline{f \times 1_{n}}, \alpha_{i} \tilde{\alpha}_{i} F(2 k)_{n} \mid i \in \underline{k}\right\rangle^{N} \\
& =\left\langle\left(\alpha_{i} \tilde{\alpha}_{i} F(2 k)_{n}\right) \overline{f \times 1_{n}}, \alpha_{i} \tilde{\alpha}_{i} F(2 k)_{n} \mid i \in \underline{k}\right\rangle^{N},
\end{aligned}
$$

that $\left(\operatorname{ker}\left(\xi_{n}\left(q_{g}\right)_{n}\right)\right) \overline{\beta_{n}}=\operatorname{ker}\left(\xi_{n}\left(q_{f}\right)_{n}\right) . \quad$ Similarly $\left(\operatorname{ker}\left(\xi_{n}\left(q_{g^{-1}}\right)_{n}\right)\right) \overline{\gamma_{n}}=\operatorname{ker}\left(\xi_{n}\left(q_{f-1}\right)_{n}\right)$, but $\operatorname{ker}\left(q_{f^{-1}}\right)_{n}=\operatorname{ker}\left(q_{f}\right)_{n}$ and $\operatorname{ker}\left(q_{g^{-1}}\right)_{n}=\operatorname{ker}\left(q_{g}\right)_{n}$. Therefore there are special isomorphisms $\overline{\overline{\bar{\beta}}}_{n}, \overline{\overline{\bar{\gamma}}}_{n}: \frac{G(\hat{g})}{G(\hat{g})_{n}} \rightarrow \frac{G(\hat{f})}{G(\hat{f})_{n}}$ making the following diagram commutative

$$
\begin{aligned}
& \frac{G(\hat{g})}{G(\hat{g})_{n}} \stackrel{\overline{\bar{\gamma}}_{n}}{\longrightarrow} \frac{G(\hat{f})}{G(\hat{f})_{n}} \\
& \left(q_{g}\right)_{n} \uparrow \quad \uparrow\left(q_{f}\right)_{n} \\
& \frac{F(k)}{F(k)_{n}} \stackrel{\overline{\bar{\gamma}}_{n}}{\longrightarrow} \frac{F(k)}{F(k)_{n}} \\
& \xi_{n} \uparrow \quad \xi_{n} \\
& \frac{F(2 k)}{F(2 k)_{n}} \stackrel{\bar{\gamma}_{n}}{\longrightarrow} \frac{F(2 k)}{F(2 k)_{n}} \\
& \overline{g \times 1}_{n} \downarrow \quad \downarrow \overline{f \times 1}_{n} \\
& \frac{F(2 k)}{F(2 k)_{n}} \stackrel{\bar{\beta}_{n}}{\longrightarrow} \frac{F(2 k)}{F(2 k)_{n}} \\
& \xi_{n} \downarrow \downarrow \xi_{n} \\
& \frac{F(k)}{F(k)_{n}} \stackrel{\overline{\bar{\beta}}_{n}}{\longrightarrow} \frac{F(k)}{F(k)_{n}} \\
& \left(q_{g}\right)_{n} \downarrow \downarrow\left(q_{f}\right)_{n} \\
& \frac{G(\hat{g})}{G(\hat{g})_{n}} \stackrel{\overline{\bar{\beta}}_{n}}{\longrightarrow} \frac{G(\hat{f})}{G(\hat{f})_{n}}
\end{aligned}
$$

Now it is enough to show that $\overline{\bar{\beta}}_{n}=\overline{\bar{\gamma}}_{n}$.

For each $i \in \underline{k}$, let $\mu_{i}=\left(\alpha_{i} F(k)_{n}\right)\left(q_{g}\right)_{n}$. Then the $\mu_{i}, i \in \underline{k}$, generate $\frac{G(\hat{g})}{G(\hat{g})_{n}}$.

From diagram (1) we have

$$
\left(\mu_{i}\right) \overline{\bar{\gamma}}_{n}=\left(\alpha_{i} F(2 k)_{n}\right) \xi_{n}\left(q_{g}\right)_{n} \overline{\bar{\gamma}}_{n}=\left(\alpha_{i} F(2 k)_{n}\right) \bar{\gamma}_{n} \xi_{n}\left(q_{f}\right)_{n} .
$$


On the other side,

$$
\left(\alpha_{i} F(k)_{n}\right)\left(q_{g}\right)_{n}=\left(\left(\alpha_{i} F(k)_{n}\right) \bar{g}_{n}\right)\left(q_{g}\right)_{n},
$$

so

$$
\begin{aligned}
\left(\mu_{i}\right) \overline{\bar{\beta}}_{n} & =\left[\left(\alpha_{i} F(k)_{n}\right)\left(q_{g}\right)_{n}\right] \overline{\bar{\beta}}_{n}=\left[\left(\alpha_{i} F(2 k)_{n}\right) \xi_{n}\left(q_{g}\right)_{n}\right] \overline{\bar{\beta}}_{n} \\
& =\left[\left(\alpha_{i} F(2 k)_{n}\right) \overline{g \times 1}_{n} \xi_{n}\left(q_{g}\right)_{n}\right] \overline{\bar{\beta}}_{n}=\left(\alpha_{i} F(2 k)_{n}\right) \bar{\gamma}_{n} \overline{f \times 1}_{n} \xi_{n}\left(q_{f}\right)_{n} .
\end{aligned}
$$

To show that $\left(\mu_{i}\right) \overline{\bar{\gamma}}_{n}=\left(\mu_{i}\right) \overline{\bar{\beta}}_{n}$ it is enough to show that for the generators $w=\alpha_{i} F(2 k)_{n}$ and $w=\tilde{\alpha}_{i} F(2 k)_{n}$ we have $\left((w) \overline{f \times 1}_{n} w^{-1}\right) \in \operatorname{ker} \xi_{n}\left(q_{f}\right)_{n}$, and then to take $w=\left(\alpha_{i} F(2 k)_{n}\right) \bar{\gamma}_{n}$.

We have seen that $\operatorname{ker}\left(\xi_{n}\left(q_{f}\right)_{n}\right)=\left\langle\left(\alpha_{i} F(2 k)_{n}\right) \overline{f \times 1_{n}} \alpha_{i}^{-1} F(2 k)_{n}, \alpha_{i} \widetilde{\alpha}_{i} F(2 k)_{n}\right|$ $i \in k\rangle^{N}$. If $w=\alpha_{i} F(2 k)_{n}$, then $(w) \overline{f \times 1_{n}} w^{-1}=\left(\alpha_{i} F(2 k)_{n}\right) \overline{f \times 1_{n}} \alpha_{i}^{-1} F(2 k)_{n} \in$ $\operatorname{ker}\left(\xi_{n}\left(q_{f}\right)_{n}\right) . \quad$ If $w=\widetilde{\alpha}_{i} F(2 k)_{n}$, then $(w) \overline{f \times 1_{n}} w^{-1}=\left(\widetilde{\alpha}_{i} F(2 k)_{n}\right) \overline{f \times 1_{n}}{\widetilde{\alpha_{i}}}^{-1} F(2 k)_{n}$, but $\left(\widetilde{\alpha}_{i} F(2 k)_{n}\right) \overline{f \times 1}_{n}=\widetilde{\alpha}_{i} F(2 k)_{n}$ since the last $k$ longitudes of $f \times 1$ are trivial and $\overline{f \times 1}_{n}$ conjugates the classes of the meridians by the correspondent longitudes (see [6]). Thus $\left(\widetilde{\alpha}_{i} F(2 k)_{n}\right) \overline{f \times 1} \tilde{\alpha}_{i}{ }^{-1} F(2 k)_{n}=1 F(2 k) n \in \operatorname{ker}\left(\xi_{n}\left(q_{f}\right)_{n}\right)$.

Therefore $\overline{\bar{\gamma}}_{n}=\overline{\bar{\beta}}_{n}$ and we have a $n$-level braid-special isomorphism as stated.

COROLlary 22. Let $f$ and $g$ be $k$-string links. If $\hat{f}$ and $\hat{g}$ are concordant, then there exists a braid-special isomorphism between the group diagram of $f$ and the group diagram of $g$.

\section{Correction}

We will take the opportunity to correct a mistake. In our paper [1] one should replace the diagram in the definition of a group diagram for the homotopy class [L] (Definition 12 there) by the commutative diagram

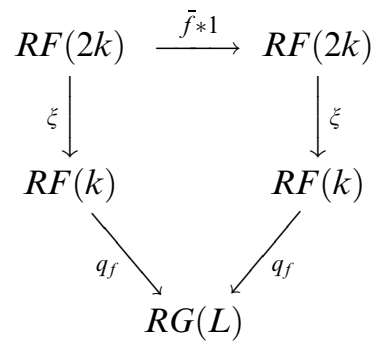

and the diagram in the definition of a braid-special isomorphism (Definition 13 there) by the commutative diagram: 


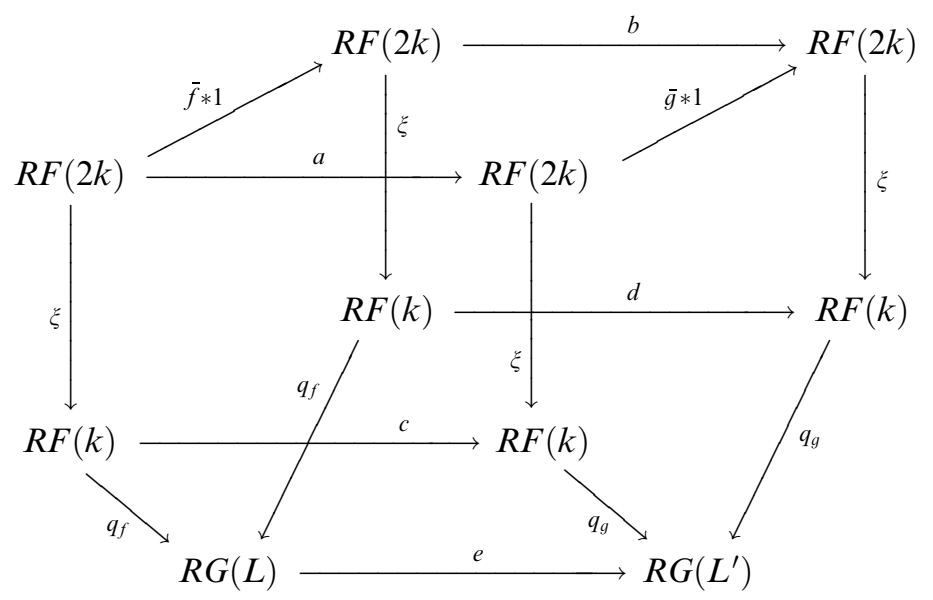

where $a, b$ are braid-like isomorphisms and $c, d, e$ are special isomorphisms.

After making these changes we will have the converse in Theorem 8 there.

\section{REFERENCES}

[1] J. E. P. P. DE CAmpos, Distinguishing links up to link-homotopy by algebraic methods, Topology Appl. 157 (2010), 605-614.

[2] J. E. P. P. DE Campos, Boundary string links, J. Knot Theory Ramifications 8 (1999), 855-878.

[3 3 S. E. Cappell and J. L. Shaneson, Link cobordism, Comment. Math. Helvetici 55 (1980), $20-49$.

[4] J. Y. Le Dimet, Cobordism d'enlacements de disques, Memoire de la Société Mathématique de France 32, Supplément au Bulletin de la S.M.F. 116, 1988.

[5] R. H. Fox and J. W. Milnor, Singularities of 2-spheres in 4-space and cobordism of knots, Osaka J. Math. (1966), 257-267.

[6] N. HabegGer and X. S. Lin, On link concordance and Milnor's $\bar{\mu}$-invariants, Bull. London Math. Soc. 30 (1998), 419-428.

[7] N. HabegGer and X. S. Lin, The classification of links up to link-homotopy, J. Amer. Math. Soc. 3 (1990), 389-419.

[8] K. H. Ko, Seifert matrices and boundary link cobordism, Trans Amer. Math. Soc. 299 (1987), 657-681.

[9] J. P. Levine, Link concordance and algebraic closure of groups, Comment. Math. Helvetici 64 (1989), 236-255.

[10] J. P. Levine, Link concordance and algebraic closure, II, Invent. Math. 96 (1989), 571-592.

[11] J. P. Levine, An approach to homotopy classification of links, Trans. Amer. Math. Soc. 306 (1988), 361-387.

[12] J. P. Levine, The $\bar{\mu}$-invariants of based links, Proceedings of the Second Topology Symposion (A. Dold and B. Eckmann, eds.), Springer-Verlag, 1987, 87-103.

[13] J. Milnor, Link groups, Ann. of Math. 59 (1954), 177-195.

[14] J. Stallings, Homology and central series of groups, J. Algebra 2 (1965), 170-181.

[15] A. G. Tristram, Some cobordism invariants for links, Proc. Cambridge Philos. Soc. 66 (1969), 251-264. 
[16] F. Waldhausen, On irreducible 3-manifolds which are sufficiently large, Ann. of Math. 87 (1968), 56-88.

José Eduardo Prado Pires de Campos

Instituto de Ciências Matemáticas e de Computação

Universidade de São Paulo

Caixa Postal 668, 13560-970

SÃo CARLOS, SP

BRAZIL

E-mail: jeppc@icmc.usp.br 